Herz 2022 $47: 175$

https://doi.org/10.1007/s00059-022-05096-9

Online publiziert: 20. Januar 2022

(C) The Author(s), under exclusive licence to Springer Medizin Verlag GmbH, ein Teil von Springer Nature 2022

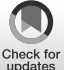

\section{Erratum zu: Diagnostik und Therapie der Lungenembolie}

Stavros Konstantinides ${ }^{1}$. Anna Mavromanoli ${ }^{1}$ Lukas Hobohm ${ }^{1,2}$

${ }^{1}$ Centrum für Thrombose und Hämostase (CTH), Universitätsmedizin der Johannes-Gutenberg-Universität Mainz, Mainz, Deutschland

${ }^{2}$ Zentrum für Kardiologie - Kardiologie I, Universitätsmedizin der Johannes-Gutenberg-Universität Mainz, Mainz, Deutschland

\section{Erratum zu:}

\section{Herz 2021}

https://doi.org/10.1007/s00059-021-

05078-3

Im oben genannten Artikel hat sich in der 0 Tab. 6 "Parenterale und orale Antikoagulanzien für die Behandlung akuter Venenthrombosen und Lungenembolien" ein Fehler bei der Dosisangabe zu Apixaban eingeschlichen. Begonnen wird nicht mit $10 \mathrm{mg} 1-\mathrm{mal} / \mathrm{Tag}$, sondern mit $10 \mathrm{mg}$ 2-mal/Tag.

Die korrigierte $\square$ Tab. 6 finden Sie hier:

Autoren und Verlag entschuldigen sich für den Fehler.

\section{Korrespondenzadresse}

Prof. Dr. Stavros Konstantinides

Centrum für Thrombose und Hämostase (CTH), Universitätsmedizin der Johannes-GutenbergUniversität Mainz

Langenbeckstraße 1, 55131 Mainz,

Deutschland

stavros.konstantinides@unimedizin-mainz.de
Die Online-Version des Originalartikels ist unter https://doi.org/10.1007/s00059-021-05078-3 zu finden.

\begin{tabular}{|c|c|c|}
\hline Substanz & \multicolumn{2}{|l|}{ Dosierung } \\
\hline \multicolumn{3}{|l|}{ Heparine } \\
\hline $\begin{array}{l}\text { Unfraktioniertes } \\
\text { Heparin (i.v.) }\end{array}$ & \multicolumn{2}{|c|}{$\begin{array}{l}\text { Beginn mit } 80 \mathrm{IE} / \mathrm{kg} \text { Bolusinjektion } \\
\text { Anschließend: } \\
\rightarrow \text { Dauerinfusion } 18 \mathrm{IE} / \mathrm{kg} / \mathrm{h} \text {, Infusionsrate an aPTT anpassen (Ziel: } 1,5 \text { - bis } \\
\text { 2,0-Faches des Normwertes) }\end{array}$} \\
\hline Enoxaparin (s.c.) & 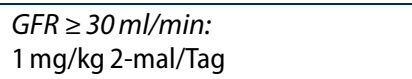 & $\begin{array}{l}\text { GFR }<30 \mathrm{ml} / \mathrm{min}: \\
1 \mathrm{mg} / \mathrm{kg} 1-\mathrm{mal} / \mathrm{Tag}\end{array}$ \\
\hline Dalteparin (s.c.) & \multicolumn{2}{|c|}{$\begin{array}{l}100 \mathrm{IE} / \mathrm{kg} \text { 2-mal/Tag oder } \\
200 \mathrm{IE} / \mathrm{kg} \text { (Höchstdosis 18.000 IE) 1-mal/Tag }\end{array}$} \\
\hline Tinzaparin (s.c.) & \multicolumn{2}{|c|}{$175 \mathrm{IE} / \mathrm{kg}$ (Höchstdosis 20.000 IE) 1-mal/Tag } \\
\hline $\begin{array}{l}\text { Fondaparinux } \\
\text { (s.c.) }\end{array}$ & $\begin{array}{l}\text { GFR } \geq 30 \mathrm{ml} / \mathrm{min}: \\
\mathrm{KG}<50 \mathrm{~kg} \rightarrow 5 \mathrm{mg} 1-\mathrm{mal} / \mathrm{Tag} \\
\mathrm{KG} 50-100 \mathrm{~kg} \rightarrow 7,5 \mathrm{mg} 1-\mathrm{mal} / \mathrm{Tag} \\
\mathrm{KG}>100 \mathrm{~kg} \rightarrow 10 \mathrm{mg} 1-\mathrm{mal} / \mathrm{Tag}\end{array}$ & $\begin{array}{l}\text { GFR }<30 \mathrm{ml} / \mathrm{min}: \\
\text { kontraindiziert }\end{array}$ \\
\hline \multicolumn{3}{|c|}{ Direkte orale Antikoagulanzien } \\
\hline Dabigatran (DTI) & \multicolumn{2}{|c|}{$\begin{array}{l}\text { Beginn mit Heparin (erste } 5-10 \text { Tage) } \\
\text { Anschließend: } \\
\rightarrow 150 \mathrm{mg} \text { 2-mal/Tag oder } \\
\rightarrow 110 \mathrm{mg} \text { 2-mal/Tag (Alter } \geq 80 \text { Jahre, gleichzeitige Verapamileinnahme) }\end{array}$} \\
\hline $\begin{array}{l}\text { Edoxaban } \\
\text { (anti-Xa) }\end{array}$ & \multicolumn{2}{|c|}{$\begin{array}{l}\text { Beginn mit Heparin (erste } 5-10 \text { Tage) } \\
\text { Anschließend: } \\
\rightarrow 60 \mathrm{mg} 1 \text {-mal/Tag oder } \\
\rightarrow 30 \mathrm{mg} 1-\mathrm{mal} / \mathrm{Tag}(\mathrm{GFR} 15-50 \mathrm{ml} / \mathrm{min}, \mathrm{KG} \leq 60 \mathrm{~kg} \text { ) }\end{array}$} \\
\hline $\begin{array}{l}\text { Apixaban } \\
\text { (anti-Xa) }\end{array}$ & \multicolumn{2}{|c|}{$\begin{array}{l}\text { Beginn mit } 10 \mathrm{mg} 2 \text {-mal/Tag (1. Woche) } \\
\text { Anschließend: } \\
\rightarrow 5 \mathrm{mg} 2 \text {-mal/Tag }\end{array}$} \\
\hline $\begin{array}{l}\text { Rivaroxaban } \\
\text { (anti-Xa) }\end{array}$ & \multicolumn{2}{|c|}{$\begin{array}{l}\text { Beginn mit } 15 \mathrm{mg} 2 \text {-mal/Tag (für } 3 \text { Wochen) } \\
\text { Anschließend: } \\
\rightarrow 20 \mathrm{mg} 1 \text {-mal/Tag oder } \\
\rightarrow 15 \mathrm{mg} 1 \text {-mal/Tag (wenn das Blutungsrisiko überwiegt) }\end{array}$} \\
\hline \multicolumn{3}{|c|}{$\begin{array}{l}\text { aPTT aktivierte partielle Thromboplastinzeit, DTI direkter Thrombininhibitor, GFR glomeruläre Filtra- } \\
\text { tionsrate, IE Internationale Einheiten, i.v. intravenös, KG Körpergewicht, s.c. subkutan, Xa aktivierter } \\
\text { Gerinnungsfaktor X }\end{array}$} \\
\hline
\end{tabular}

